\title{
Phase Advancement and Nucleus-Specific Timing of Thalamocortical Activity during Slow Cortical Oscillation
}

\author{
Andrea Slézia, ${ }^{1 *}$ Balázs Hangya, ${ }^{2 *}$ István Ulbert, ${ }^{3,4}$ and László Acsády ${ }^{1}$ \\ ${ }^{1}$ Laboratory of Thalamus Research, Institute of Experimental Medicine and ${ }^{2}$ Laboratory of Cerebral Cortex Research, Institute of Experimental Medicine, \\ Hungarian Academy of Sciences, 1083, Budapest, Hungary, ${ }^{3}$ Institute for Psychology, Hungarian Academy of Sciences, 1068, Budapest, Hungary, and ${ }^{4}$ Péter \\ Pázmány Catholic University, Faculty of Information Technology, 1083, Budapest, Hungary
}

The exact timing of cortical afferent activity is instrumental for the correct coding and retrieval of internal and external stimuli. Thalamocortical inputs represent the most significant subcortical pathway to the cortex, but the precise timing and temporal variability of thalamocortical activity is not known. To examine this question, we studied the phase of thalamic action potentials relative to cortical oscillations and established correlations among phase, the nuclear location of the thalamocortical neurons, and the frequency of cortical activity.

The phase of thalamic action potentials depended on the exact frequency of the slow cortical oscillation both on long (minutes) and short (single wave) time scales. Faster waves were accompanied by phase advancement in both cases. Thalamocortical neurons located in different nuclei fired at significantly different phases of the slow waves but were active at a similar phase of spindle oscillations. Different thalamic nuclei displayed distinct burst patterns. Bursts with a higher number of action potentials displayed progressive phase advancement in a nucleus-specific manner. Thalamic neurons located along nuclear borders were characterized by mixed burst and phase properties.

Our data demonstrate that the temporal relationship between cortical and thalamic activity is not fixed but displays dynamic changes during oscillatory activity. The timing depends on the precise location and exact activity of thalamocortical cells and the ongoing cortical network pattern. This variability of thalamic output and its coupling to cortical activity can enable thalamocortical neurons to actively participate in the coding and retrieval of cortical signals.

\section{Introduction}

The role of the thalamus is traditionally discussed in two contexts, sensory information transmission and thalamocortical oscillations (Steriade et al., 1997). In the case of sensory relay, the timing of thalamic firing is largely determined by the stimulus parameters (Sherman and Guillery, 2006). Timing in case of oscillatory activity is less obvious. In this latter context, thalamic activity is often described as an autonomous pacemaker: "the thalamic clock" (Buzsáki, 1991; Steriade et al., 1993b; Contreras and Steriade, 1997). Implicit in the clock hypothesis is that the temporal relationship between thalamic and cortical activity is fixed and all neurons beat the same rhythm. The alternative hypothesis would predict that the thalamic activity is variable in time or possibly across specific cell populations. In the cortex (e.g., in the hippocampus), the phase relationship between neu-

Received June 30, 2010; revised Sept. 3, 2010; accepted Sept. 20, 2010.

This work was supported by the Wellcome Trust (L.A. is in receipt of a Wellcome Project Grant), the Hungarian Scientific Research Fund (Grants OTKA T 75676 and OTKA K 81357), and the National Office for Research and Technology-French National Research Agency TéT Fund (NKTH-Neurogen). We thank Krisztina Faddi for her excellent technical assistance. We thank Hajnalka Bokor, Péter Barthó, Viktor Varga, Szabolcs Káli, Ferenc Mátyás, Viktor M. Plattner, Zita Rovó, Mark D. Eyre, and Attila Kaszás for their helpful comments.

${ }^{*}$ A.S. and B.H. contributed equally to this work.

Correspondence should be addressed to László Acsády, Institute of Experimental Medicine, Hungarian Academy of Sciences, P.0. Box 67, 1450, Budapest, Hungary. E-mail: acsady@koki.hu.

DOI:10.1523/JNEUROSCI.3375-10.2011

Copyright $\odot 2011$ the authors $\quad 0270-6474 / 11 / 310607-11 \$ 15.00 / 0$ ronal activity and the local field potential (LFP) is variable (phase precession) (O'Keefe and Recce, 1993), and distinct neuronal classes fire at different phases of the local oscillation (Freund and Buzsáki, 1996; Klausberger and Somogyi, 2008). Consistent phase shifts, or population-specific timing, however, have not been detected in the thalamus. Thus, in this study, we examined the dynamics of thalamocortical timing by analyzing the phase and nucleus specificity of thalamic action potential generation relative to cortical oscillations. The phase of relay cell firing was determined in four contexts.

First, the relationship between the exact frequency of a given cortical oscillation and the phase of thalamic activity was examined. Second, the nucleus specificity of the phase was determined, because the thalamus is known to contain different nuclei, which receive distinct inputs and target distinct layers and dendritic domains in the cortex (Herkenham, 1980; Jones, 1985). Because boundaries between thalamic nuclei are not sharp, well defined edges, special attention was focused on the physiological properties of neurons located along the border zones between nuclei. Third, the relationship between the exact firing pattern and the timing of activity was analyzed. Thalamocortical cells are known to display different types of bursting activity (Jahnsen and Llinás, 1984). Bursts containing a different number of action potentials have distinct effects on cortical neurons (Swadlow and Gusev, 2001). Thus, the interaction between phase and the number of action potential within a burst was determined. Finally, thalamo- 
cortical circuits can display different types of oscillations, with each member of the circuit playing distinct roles during various oscillatory patterns (Llinás and Steriade, 2006). Thus, we examined the interaction among the type of oscillation (slow oscillation vs spindle activity), location, and phase.

The experiments were performed using rats anesthetized with ketamine/xylazine in the two best characterized somatosensory nuclei: the first order ventrobasal complex [VB, containing the ventral posteromedial nucleus (VPM) and ventral posterolateral nucleus (VPL)] and the higher order posterior nucleus (Po) (Yu et al., 2006; Diamond et al., 2008). Our choice of nuclei was based on the fact that VB and Po receive distinct types of cortical inputs (Hoogland et al., 1991). Using this model system, we describe highly specific spatial and temporal inhomogeneity in thalamocortical activity.

\section{Materials and Methods}

Anesthesia and surgery. Thirty-four adult male Wistar rats (300-400 g) were used for the experiments. All experimental procedures were performed according to the ethical guidelines of the Institute of Experimental Medicine of the Hungarian Academy of Sciences and approved by the Ethical Committee. Surgery and experiments were done under ketamine/xylazine anesthesia. Initially, rats received an intraperitoneal injection of ketamine $(83 \mathrm{mg} / \mathrm{kg})$ and xylazine $(3.3 \mathrm{mg} / \mathrm{kg})$. For the maintenance of the anesthesia, intramuscular injections of ketamine/ xylazine were given every $30-50 \mathrm{~min}$ during the entire period of the experiments.

LFP recording and in vivo juxtacellular recording and labeling. For LFP recordings, rats were implanted with monopolar surface electrodes (stainless steel screw, diameter: $1 \mathrm{~mm}$, length: $2 \mathrm{~mm}$ ) in the primary somatosensory cortex (S1, L: $-5.5 \mathrm{~mm}$, AP: $-1.5 \mathrm{~mm}$ from bregma according to Paxinos and Watson, 1998). The recorded signal was amplified, bandpass filtered $(0.1 \mathrm{~Hz}$ to $5 \mathrm{kHz}$; Supertech BioAmp, Supertech), and digitized at $16.6 \mathrm{kHz}$ (micro 1401 mkii, CED). Unit activity was recorded by glass microelectrodes (in vivo impedance of $20-40 \mathrm{M} \Omega$ ) pulled from borosilicate glass capillaries $(1.5 \mathrm{~mm}$ outer diameter, 0.75 or 0.86 inner diameter, Sutter Instrument or WPI) and filled with $0.5 \mathrm{M}$ $\mathrm{NaCl}$ and 2\% neurobiotin (Vector Laboratories). Electrodes were lowered by a piezoelectric microdrive (Burleigh 6000 ULN or ISS 8200 , EXFO) into the somatosensory thalamus (L: $2.8 \mathrm{~mm}, \mathrm{P}: 3.3 \mathrm{~mm}, \mathrm{~V}$ : $4.8-6.8 \mathrm{~mm}$ from bregma). Neuronal signals were amplified by a DC amplifier (Axoclamp 2B, Molecular Devices), further amplified, and filtered between 0.16 and $5 \mathrm{kHz}$ by a signal conditioner (LinearAmp, Supertech). Neuronal signals recorded by Spike2 5.0 (CED) software were upsampled at $20 \mathrm{kHz}$ for mathematical analysis.

Juxtacellular labeling of the recorded neurons was done as previously described (Pinault, 1996). Briefly, positive current was injected through the recording electrode using a $200 \mathrm{~ms}$ on-off duty cycle on top of lowintensity negative DC current $(0-0.4 \mathrm{nA})$. The activity of the neurons was continuously monitored, and the intensity of the injected current was increased until the modulation of neuronal firing was achieved (up to $19 \mathrm{nA}$, for $1-15 \mathrm{~min}$ ).

Histology and immunocytochemistry. Following labeling and a survival period (from $15 \mathrm{~min}$ to $2 \mathrm{~h}$ ), animals were transcardially perfused first with saline (maximum $2 \mathrm{~min}$ ), then with $400-500 \mathrm{ml}$ of fixative containing $4 \%$ paraformaldehyde, $0.05 \%$ glutaraldehyde in $0.1 \mathrm{M}$ phosphate buffer. Tissue blocks were cut on a Vibratome into $50 \mu \mathrm{m}$ coronal sections. Following freeze-thawing, the sections were incubated with avidin-biotin complex (ABC, Vector Laboratories; 1:300) in Tris-buffered saline (TBS) for $2 \mathrm{~h}$ and were developed using nickel intensified diaminobenzidine as a chromogen for the visualization of labeled neurons.

Vesicular glutamate transporter type 2 (vGluT2) immunostaining was used to establish the precise nuclear boundary between VPM and Po (see Fig. $4 E$ ). Three different vGluT2 antisera raised against different epitopes of the vGluT2 molecule were used to determine the specificity of the immunostaining. These were rabbit anti-vGluT2 (1:3000, Synaptic Systems), guinea pig anti-vGluT2 (1:3000, Millipore Bioscience Research
Reagents), and mouse anti-vGluT2 (1:3000, Millipore Bioscience Research Reagents). vGluT2 was visualized using the corresponding biotinylated secondary antibodies, $\mathrm{ABC}$ and $\mathrm{DAB}$, as a chromogen, which resulted in a brown reaction product. Immunoreaction with the three vGluT2 antisera resulted in identical immunostaining in the thalamus. The sections were then osmicated, dehydrated, and flat-embedded in Durcupan for light microscopic analysis. The osmium solution contained 7\% sucrose to preserve color difference.

Photomicrographs were taken with a Spot RT camera (Diagnostic Instruments) or with an AxioCam HRC (Carl Zeiss Microimaging). Digital montages of serial photos taken through the thickness of a section were processed with the "extended depth of field function" of Image-Pro Express 6.0 (Media Cybernetics). When necessary, brightness and contrast were adjusted using Adobe Photoshop CS2 (Adobe Systems) applied to whole images only.

In the case of two border cells, three-dimensional reconstruction of the dendritic tree and the soma was performed using Neurolucida 5.2 software (MBF Bioscience).

Data analysis. Raw data were imported to the Matlab development environment (MathWorks) for analysis using built-in and customwritten functions.

Burst detection and burst analysis. Instead of using arbitrary intra- and interburst parameters for burst detection, a custom-built semiautomatic burst detection method was used. Interspike intervals (ISIs) were clustered using Ward's amalgamation rule and Euclidean metric, assuming that the ISI cluster containing the shortest interval corresponds to the intraburst intervals. Clustering was iteratively performed by varying the number of clusters from 2 to the theoretical maximum. Minimal intraburst frequency was displayed in the function of the number of clusters. Instead of determining fixed limiting values for the above parameter, implausibly low values $(\sim 80 \mathrm{~Hz})$ were used as exclusion parameters. The largest nonexcluded step in the minimal intraburst frequency function was determined and the clustering corresponding to the cluster number after that step was chosen. Bursts were verified post hoc by visual examination, and in a few cases the chosen step was modified to a neighboring step in the minimal intraburst frequency function (supplemental Fig. 1, available at www.jneurosci.org as supplemental material). Because the last intraburst ISIs can be longer than some extraburst intervals, a histogram of the first ISIs after bursts was plotted for each neuron. In cases where a clear peak of short intervals could be separated $(<15 \mathrm{~ms})$, representing the last spike of the burst, these short intervals were added to the bursts (see supplemental Notes, available at www.jneurosci.org as supplemental material).

After the identification of bursts (Borhegyi et al., 2004; Varga et al., 2008), the following firing parameters were determined: firing frequency, frequency of the first action potential of the bursts (burst frequency), the number of all action potentials in bursts relative to the number of all spikes fired (burstiness index), average duration of bursts measured in milliseconds (burst length), the firing frequency for the intraburst action potentials (intraburst frequency), and the average number of spikes in bursts (intraburst spike number). Group comparisons were performed using Mann-Whitney $U$ tests and the significance of the differences was judged at the level of $p=0.05$.

The slow oscillation frequency for the LFP recordings was calculated in 30 -s-long epochs as the reciprocal of the mean slow oscillation cycle length derived from the analytic signal (see below). This allowed us to eliminate the distortion of the frequency measure by short episodes of substantially perturbed, diminished, or completely ceased slow-wave activity. To ensure that cyclic low-frequency activity was present in all segments included in the analysis, wavelet spectra, Fourier spectra, and LFP autocorrelations were calculated and visualized. For the comparison of VB and Po neurons, only the epochs with an LFP frequency between 1.1 and $1.6 \mathrm{~Hz}$ were analyzed (see Results). For the analysis of long ( $>20 \mathrm{~min}$ ) recordings, no frequency restrictions were applied when analyzing the correlation between slow oscillation frequency and firing characteristics.

Phase analysis. During deep sleep, the neocortex is characterized by slow oscillation. This can be divided into up states (the active phase), which can be recorded as a depth positive, surface negative wave, and a down state with opposite polarity. The phases of action potentials relative 


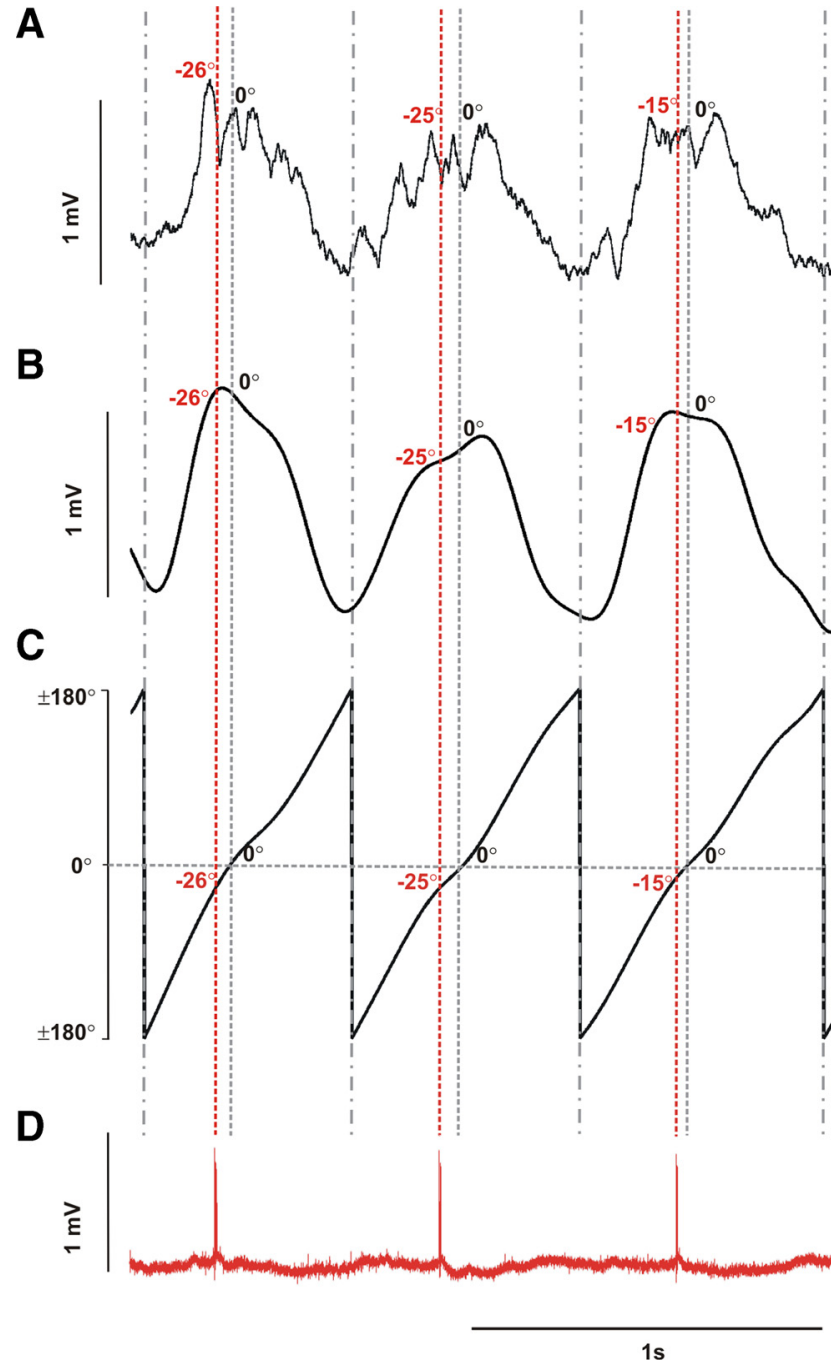

Figure 1. The method to determine the phase of thalamocortical activity relative to the cortical oscillation. $\boldsymbol{A}, \boldsymbol{B}$, The raw cortical LFP trace $(\boldsymbol{A})$ is filtered by a low-pass filter at $5 \mathrm{~Hz}(\boldsymbol{B})$. Three oscillation cycles are shown. $\boldsymbol{C}$, The Hilbert transformation is performed on the filtered trace, and the instantaneous phase is calculated (see Materials and Methods). D, The phase of thalamocortical unit activity is determined by the instantaneous phase of the LFP trace (red dashed line in $\boldsymbol{A}-\boldsymbol{C}$ ). Zero degrees corresponds to the peak of the up state (gray dashed lines), $\pm 180^{\circ}$ refers to the trough of the oscillation (gray dash-dot line). Negative phase values refer to the early, rising phase of the up state. Red numbers indicate the phase of thalamocortical activity during the three cycles.

to slow oscillation cycles in the LFP were determined by the angle of the analytic signal (also called the instantaneous phase, derived from a Hilbert transformation of the LFP) at the location of spikes (Fig. 1) (Hurtado et al., 2004). In our particular case, the peak of the up state is defined as $0^{\circ}$ after Hilbert transformation. Accordingly, the peak of the down state is $180^{\circ}$. These two points define the reference frame, which enables us to compare waves with different shapes and lengths. The absolute beginning and end of the up state is not determined, because thalamic activity is compared with each other using $0^{\circ}$ as a reference point. This method avoids defining the length, beginning, and end of the up state, which is always hotly debated and not necessary to answer our questions. Figure 1 illustrates phase analysis. The LFP was preprocessed using a zero phaseshift, low-pass, finite impulse response filter with a cutoff frequency of 5 Hz. To standardize slow oscillation frequency across animals, halfminute segments with a frequency between 1.1 and $1.6 \mathrm{~Hz}$ were selected to describe the nucleus specificity of phase. Slow-wave cycles shorter than $250 \mathrm{~ms}$ (corresponding to a $4 \mathrm{~Hz}$ upper limit) as well as cycles with LFP amplitudes lower than mean LFP +2 SD were discarded from the anal-
A1

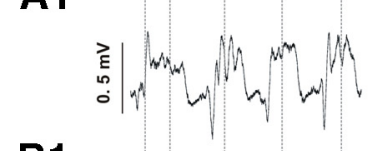

B1

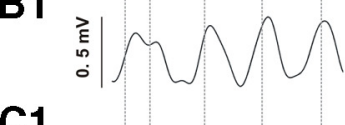

C1

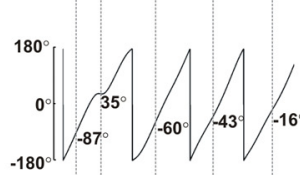

D1

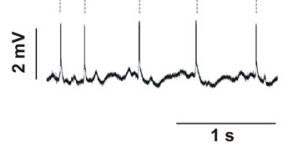

B2

A2

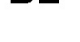

C2

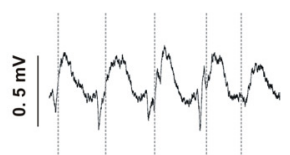

C2

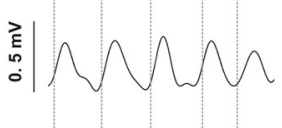

$180^{\circ}$

D2

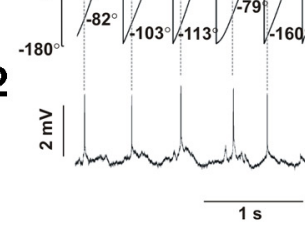

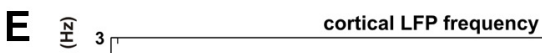

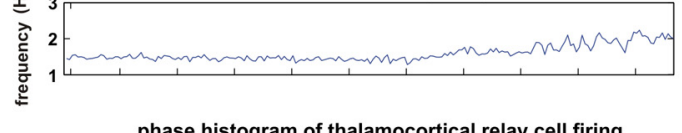

$\mathbf{F}$

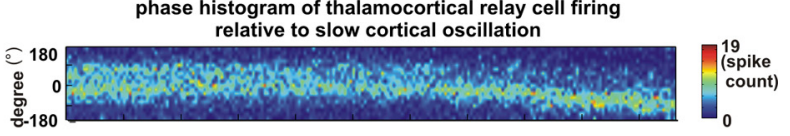

G

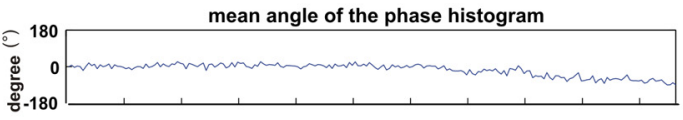

H

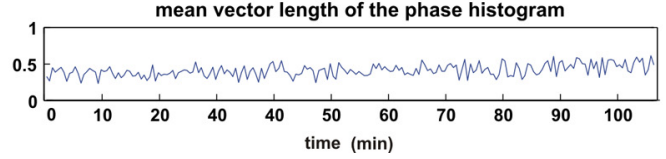

Figure 2. Phase advancement of thalamocortical activity with increasing frequency of cortical oscillation. $\boldsymbol{A 1}$-D2, $\operatorname{Raw}(\boldsymbol{A})$ and filtered $(\boldsymbol{B})$ cortical LFP trace, instantaneous phase $(\boldsymbol{C})$, and thalamocortical activity $(\boldsymbol{D})$ relative to the slow cortical oscillation from the first $(\boldsymbol{A 1} \mathbf{1}-\boldsymbol{D} \mathbf{1})$ and last (A2-D2) 30 s of a $106.5 \mathrm{~min}$ recording. Note that faster waves (A2-C2) at the end of the recording are accompanied by an earlier phase of the thalamocortical cell than slower waves of the first epoch (compare numbers referring to the phase of firing in $\mathbf{C}$ and $\mathbf{C 2}$ ). $\boldsymbol{E}$, Frequency of the LFP recorded from the $S 1$ cortex during entire period of $106.5 \mathrm{~min}$. The LFP activity remained in the delta frequency range $(1-3 \mathrm{~Hz})$ but became faster during the last third of the period. $\boldsymbol{F}$, Phase histogram of a thalamocortical neuron during the same period calculated in $30 \mathrm{~s} \mathrm{time}$ windows. Warmer colors indicate higher spike counts. Note the phase advancement of the thalamocortical cell firing in parallel to the increase of LFP frequency. The effect is quantified in $\mathbf{G}$ (mean phase angle as a function of time). The mean phase appears progressively earlier. The phase advancement was accompanied by a slight increase in the phase coupling strength $(\boldsymbol{H}$, mean vector length).

ysis. Phase angles were taken at the location of first spikes of bursts and at the location of single spikes; thus, bursts were considered as single events. Phase histogram, mean angle, and mean vector length were calculated using circular statistical methods for each recording (Fisher, 1993). Mean phase histograms were calculated by pooling 100 element random samples from the individual distributions. Only neurons that fired at least 100 action potentials during the appropriate segments were included. For the analysis of burst phases with a fixed number of action potentials, a minimal criterion of 10 bursts and 4 cells was chosen for each category. Minimal numbers were chosen to ensure statistically reliable estimates of firing phase (Fisher, 1993). Mean phase values were compared by a statistical test according to Fisher (Fisher, 1993). The comparison of phase consistency was performed by a permutation test on the phase concentration $(\kappa)$ values (Monte Carlo randomization, 10,000 permutations). Significance was judged at a level of $p=0.05$.

Action potential phase values relative to the spindle frequency oscillation were calculated by applying the Hilbert transformation method on 
the LFP filtered between 7 and $20 \mathrm{~Hz}$. This choice of frequency band boundaries was based on the localization of the power peak in the spectra. Oscillation cycles shorter than 50 ms (corresponding to a $20 \mathrm{~Hz}$ upper limit) as well as those cycles with an LFP amplitude smaller than the mean LFP +2 SD were discarded. Only neurons with significant phase coupling to spindles were processed further $(p<0.01$, Rayleigh's test). Phase distributions and related measures were computed and processed similarly to those relative to the slow oscillation (see above).

\section{Results}

\section{The phase of relay cell firing depends} on the exact frequency of cortical slow oscillation

Ketamine/xylazine anesthesia results in a relatively stable oscillation in the delta (1-3 Hz) frequency range. In this study, we refer to this activity as "slow oscillation", because its frequency is within the range that characterizes human slowwave sleep (Huber et al., 2004; Massimini et al., 2009).

During long recordings, the exact frequency of the oscillation changed over time, influenced by the depth of anesthesia. Long recordings (20-106 min) were performed in the somatosensory thalamus (Po and VB) to acquire a sufficient number of action potentials for phase analysis in the $1-3 \mathrm{~Hz}$ frequency range $(n=6)$ (Fig. 2 ). The phase of thalamocortical activity was calculated using time windows of a $30 \mathrm{~s}$ duration. The correlation between the exact frequency of cortical activity and the phase of thalamocortical firing relative to the cortical oscillation was determined. In five of six neurons, increases in the frequency of cortical oscillations resulted in a phase advancement of the firing activity (Fig. 2 ). The amount of phase advancement varied between 20 and $141^{\circ}$ per $1 \mathrm{~Hz}$ increase in the frequency of cortical oscillations (mean: $68.23^{\circ}$ ). In the remaining case, the activity became biphasic as the frequency increased, prohibiting a direct comparison of this cell with the others for this parameter.

\section{Instantaneous phase correlation during the slow oscillation}

As shown above, the phase of the thalamic action potentials was influenced by the frequency of the ongoing slow oscillation (Fig. 2 ). This can be explained either by slow influences (metabotropic or tonic mechanisms where the time course of action is too slow for wave-to-wave timing of action potentials) or possibly by fast wave-to-wave effects. To gain insight into instantaneous phase modulation, we selected individual waves with a frequency faster or slower than the average frequency of the ongoing oscillation. In 10 relay neurons, we identified individual faster waves $(2.0-$ $2.5 \mathrm{~Hz})$ during slower epochs $(1.1-1.6 \mathrm{~Hz})$ and compared the phase of firing during these faster waves with the phase measured during the regular slow waves $(1.1-1.6 \mathrm{~Hz})$ of the same epochs. Similarly, we selected individual slower waves occurring during faster epochs $(2.0-2.5 \mathrm{~Hz}, n=6)$ and compared the phase of firing during the faster and slower waves of the faster epochs.

The appearance of individual faster waves $(2.0-2.5 \mathrm{~Hz}) \mathrm{em}-$ bedded into slower $(1.1-1.6 \mathrm{~Hz})$ epochs were paralleled by immediate and significant phase advancement of thalamic unit firing $(p=0.0138$ ) (Fig. $3 A-D)$. Similarly, individual slow waves $(1.1-1.6 \mathrm{~Hz})$ during faster cortical oscillation epochs $(2.0-2.5$ $\mathrm{Hz}$ ) were accompanied by an instantaneous and significant phase delay in thalamic unit firing $(p=0.0131)$ (Fig. $3 E-H)$. On average, the degree of advance during individual fast waves vs delay during individual slow waves was in a similar range $\left(+18.85^{\circ}\right.$ vs $-25.65^{\circ}$ ) (Fig. $3 I$ ).

These results indicate that the precise timing of relay cell activity is determined not only on the longer time scale (probably reflecting tonic/metabotropic influences) but in a wave-to-wave manner as well. The direction of change was consistent in both time scales, i.e., faster waves correlated with phase advancement and slower waves correlated with phase delay.

The properties of burst activity display nuclear specificity Following the identification of frequency dependence, we looked at the dependence of relay cell firing between thalamic nuclei. Thirtyseven relay cells were recorded for this analysis. Unequivocal identification of the recorded cell and its location was established in 20 cases. In the remaining 17 cells, the location of each recorded neuron soma in a given thalamic nucleus was determined by the depth measurements of histologically identified neurons recorded in the same electrode track or in another track of the same animal (Fig. 4A). Neurons with equivocal thalamic nucleus locations were discarded. Figure $4 B$ shows examples of relay cell firing from all examined anatomical groups relative to cortical LFP.

To determine the exact nuclear position of the labeled cells, vGluT2 immunostaining was performed (Fig. 4C-E). vGluT2 labels the excitatory terminals of axons arriving from subcortical centers (Fremeau et al., 2001; Land et al., 2004; Lavallée et al., 2005). The boundary between the first order VPM and the higher order Po could be established based on denser (Fig. 4D) and larger vGluT2-positive terminals in VPM innervating the more proximal parts of relay cells. The boundary formed by 
A

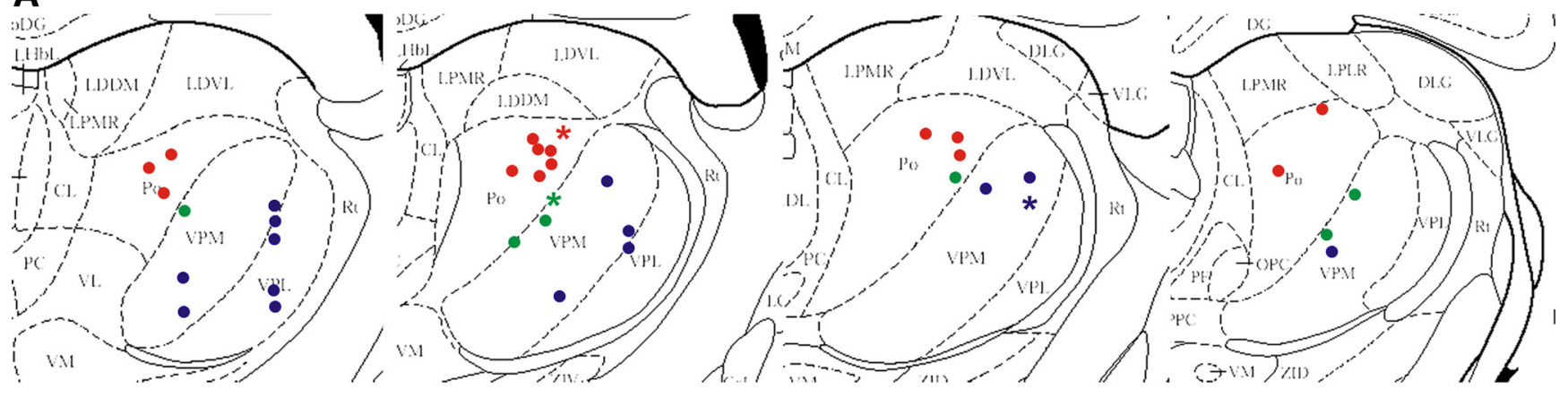

B
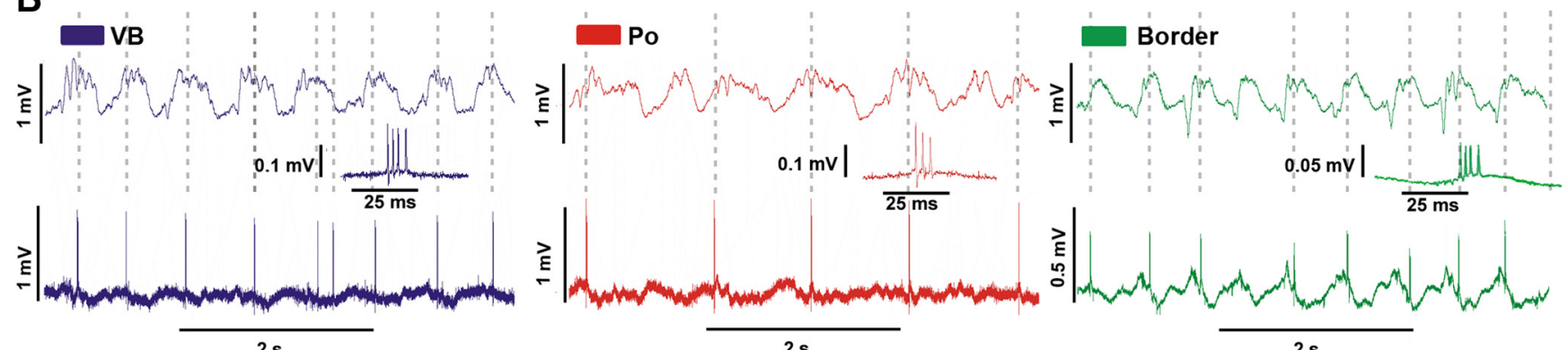

$2 \mathrm{~s}$
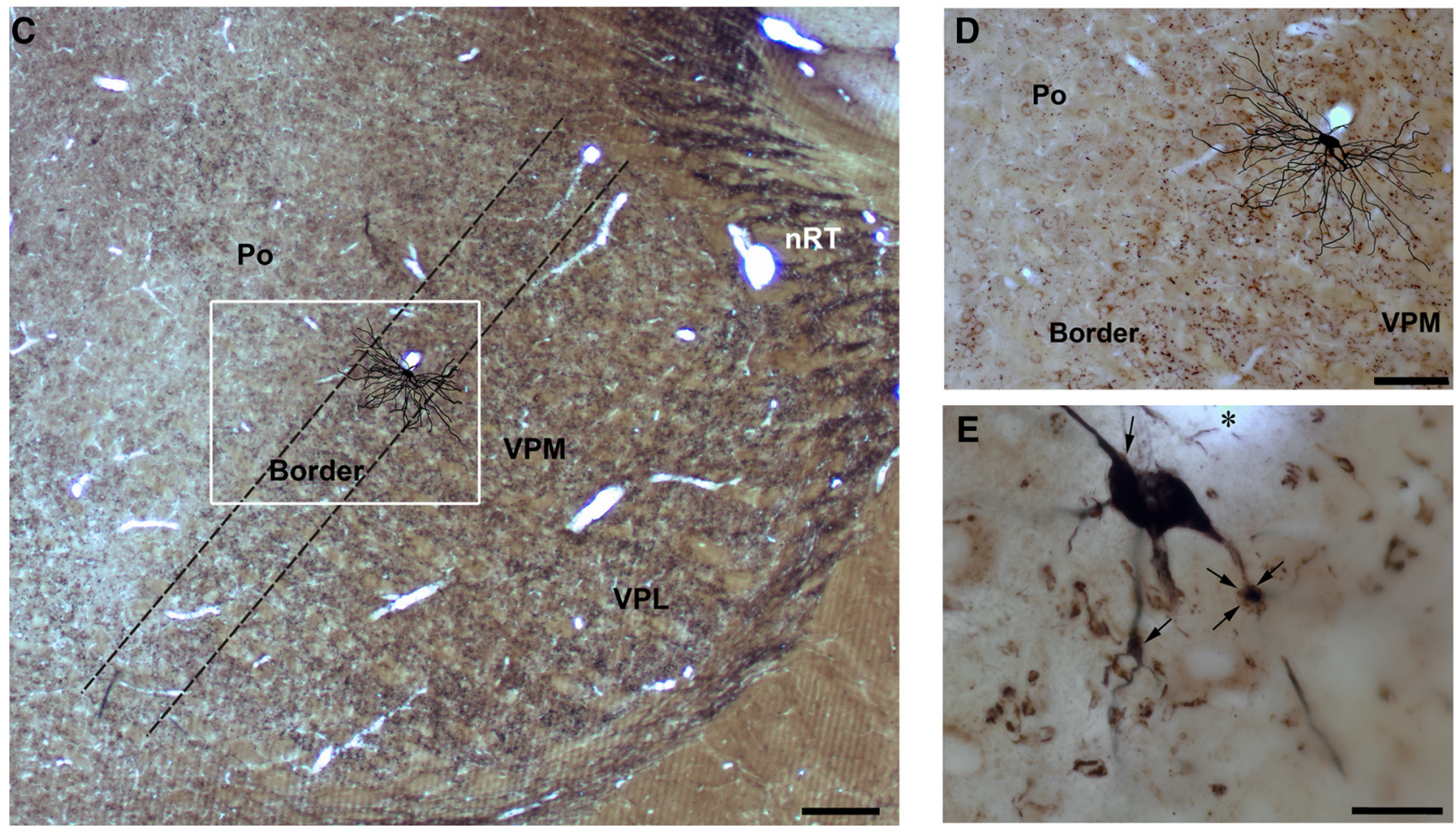

Figure 4. Nuclear localization and firing pattern of the recorded relay cells. $A$, Localization of all juxtacellularly recorded cells in the thalamus in four coronal planes according to the rat brain atlas of Paxinos and Watson (1998). Blue, red, and green dots represent the position of the recorded VB, $\mathrm{P} 0$, and Border neurons, respectively. The green asterisk indicates the neuron shown in $\boldsymbol{C}-\boldsymbol{E}$. $B$, Single unit activity of representative VB (blue), Po (red), and Border (green) cells (marked by asterisks in $\boldsymbol{A}$ ) and the simultaneously recorded LFP from the S1 cortex. Insets show burst events at a faster time scale. $C$, Neurolucida drawing of a border cell overlaid on the low-power image of the vGluT2 immunostained coronal section in which the cell body was found. The green trace in $\boldsymbol{B}$ shows juxtacellular recording of the same cell. The border region is indicated by two dashed lines in $\boldsymbol{C}$. $\boldsymbol{D}$, The inset of $\boldsymbol{C}$ (white rectangle) at higher magnification. Note the difference in vGluT2 immunostaining between Po and VPM. $\boldsymbol{E}$, At high power, large vGluT2 terminals contact the proximal dendrites of the labeled cell (arrows). Asterisk $=$ blood vessel. Scale bars: $\mathbf{C}$ and $\boldsymbol{D}, 200 \mu \mathrm{m}$; E, $20 \mu \mathrm{m}$.

vGluT2 coincided with the one determined by cytochrome oxidase and parvalbumin staining (Paxinos and Watson, 1998; Veinante et al., 2000) but was more distinct.

A 100- $\mu \mathrm{m}$-thick band on both sides of the Po-VPM border was treated separately, and the labeled cells with somata located in this zone were analyzed as a distinct group, referred to as Border cells (Fig. 4C). Neurons in this area extended dendrites into both nuclei (Fig. 4D). The $100 \mu \mathrm{m}$ limit was based on previous data in cats indicating that large excitatory terminals make contact with relay cells up to $100 \mu \mathrm{m}$ from the soma (Liu et al., 
1995), which was also confirmed by our observations in rats. Seven neurons were recorded in this Po-VPM border zone, all of which were recovered and unequivocally identified. Fifteen VB and 15 Po cells represented first and higher order relays in our sample, respectively.

Before analyzing the phase of firing, we compared the properties of bursts in the three anatomical groups. All firing properties were most stable in the $1.1-1.6 \mathrm{~Hz}$ range. Thus, we used this frequency window to test nucleus-specific activity.

Relay cells in all three groups fired regularly during the slow cortical oscillations, and their activity was coupled with the rhythmic LFP signal (Fig. 4B). The average firing frequency of $\mathrm{VB}$ and Border cells was similar (5.82 vs $6.24 \mathrm{~Hz}$ ), whereas Po neurons were significantly slower than Border neurons (3.88 Hz; Border vs Po: $p<0.001$ ) (Fig. 5A; supplemental Table 1, available at www.jneurosci.org as supplemental material). The frequency of burst events was similar between VB and Border cells, whereas Po neurons displayed a significantly lower number of bursts (Border vs Po: $p<0.001$ ) (Fig. 5B; supplemental Table 1, available at www.jneurosci.org as supplemental material). The vast majority of all action potentials (90-95\%) occurred within bursts in all three groups (Fig. 5C; supplemental Table 1, available at www.jneurosci.org as supplemental material).

The average intraburst frequency of VB and Border cells was nearly identical ( 423.16 vs $418.72 \mathrm{~Hz}$ ), whereas the bursts of Po cells were $\sim 80 \mathrm{~Hz}$ slower (341.68 $\mathrm{Hz}$ ) (VB vs Po: $p<0.001$; Border vs Po: $p<0.01$ ) (Fig. 5D; supplemental Table 1, available at www.jneurosci.org as supplemental material). The number of spikes/ bursts displayed comparable values for VB and Border cells (3.69 vs 3.77), whereas Po cells fired on average one spike less for every burst (2.67) (VB vs Po and Border vs Po: $p<$ 0.05 ) (Fig. 5E; supplemental Table 1, available at www.jneurosci.org as supplemental material). In these latter two parameters, VB and Border cells were not statistically different from each other, but they both differed significantly from Po cells (at least $p<0.05$ ).

Finally, we examined the ISI distributions in bursts with different spike numbers (supplemental Fig. 2, available at www.jneurosci.org as supplemental material). Bursts with larger spike numbers were characterized by shorter first ISIs. Within a burst, later ISIs tended to be longer than earlier ones. These features were present in all three groups examined and confirmed earlier in vitro and in vivo data (Hughes et al., 2004). The ISIs of Po cells were longer at all serial order positions than those of VB and Border cells (supplemental Fig. 2, available at www.jneurosci.org as supplemental material).
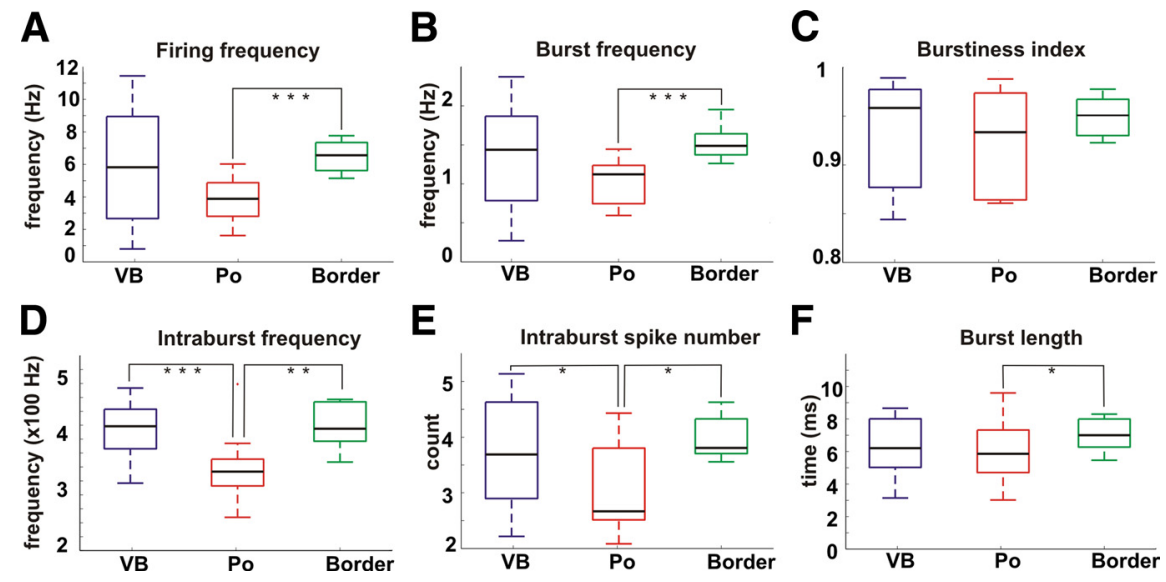

Figure 5. Firing characteristics and burst properties of $\mathrm{VB}, \mathrm{P} 0$, and Border cells. $\boldsymbol{A}-\boldsymbol{F}$, Firing properties $(\boldsymbol{A}-\boldsymbol{C})$ and burst properties ( $\boldsymbol{D}-\boldsymbol{F}$ ) of VB (blue), Po (red), and Border (green) cells. The plots display median (horizontal black line), interquartile range (box), and the range of nonoutliers (whiskers). Po neurons show a significantly lower firing rate than Border cells $(\boldsymbol{A}, \boldsymbol{B})$. The intraburst frequency and spike number of Po neurons are also significantly lower than those of VB and Border cells $(D, E)$. ${ }^{*} p<$ $0.05 ;{ }^{* *} p<0.01,{ }^{* * *} p<0.001$.
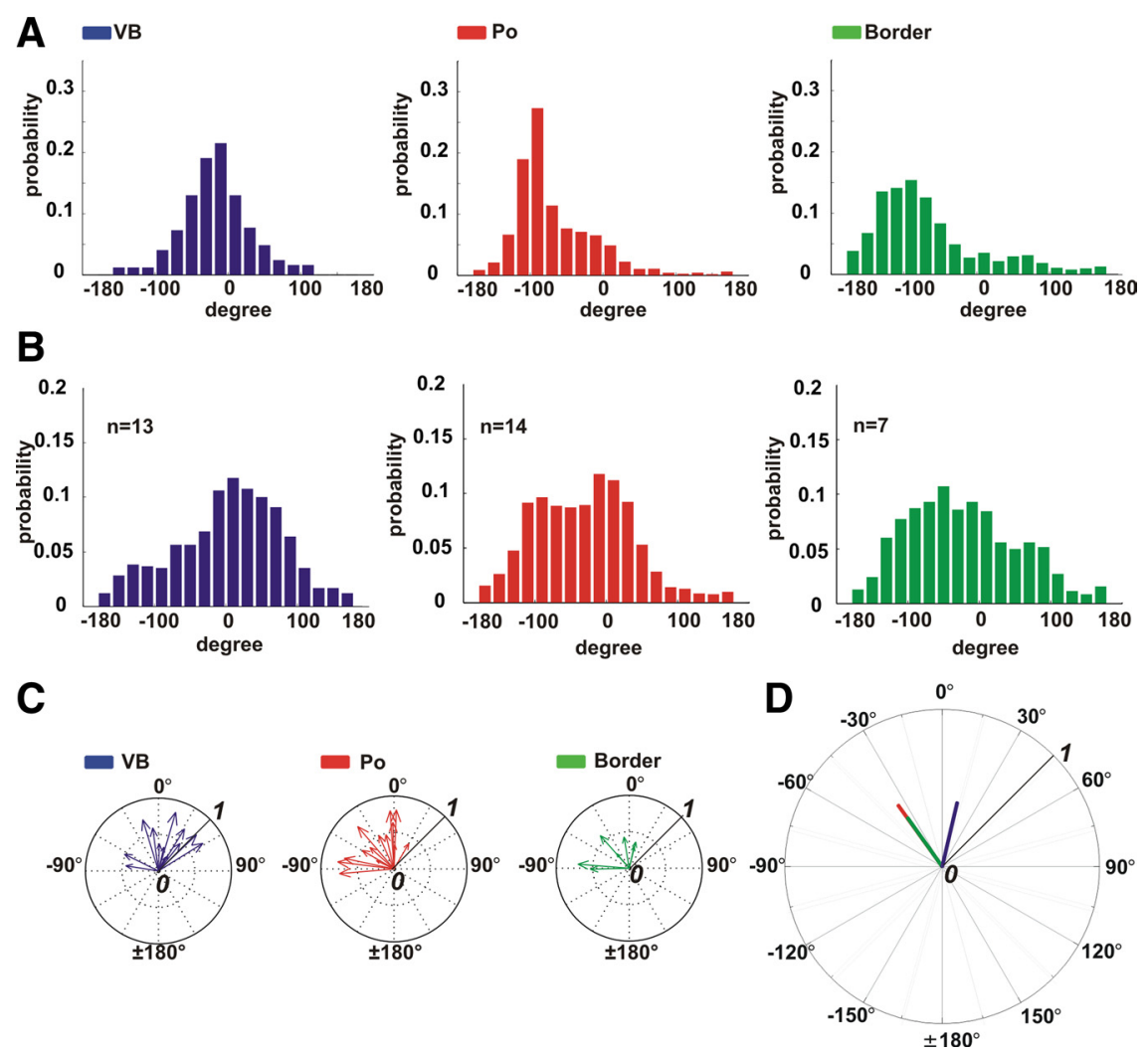

Figure 6. Nuclear specificity of phase preference. $\boldsymbol{A}-\boldsymbol{C}$, Individual phase histograms of representative VB $(\boldsymbol{A}), \mathrm{Po}_{0}(\boldsymbol{B})$, and $\mathrm{B}$ order (C) neurons. Zero degrees corresponds to the peak of the cortical up states. $\boldsymbol{B}$, Average population phase histograms of VB, $P_{0}$, and Border cells. C, Polar plots showing the phase of all individual cells. Each arrow represents a neuron of the given cell group. Note that most $\mathrm{Po}$ and Border cells fire before $0^{\circ}$, whereas most VB cells are active after the peak. $\boldsymbol{D}$, Polar plot showing the average phase vector for the three cell populations relative to slow cortical oscillations. Note that the population vector of Po and Border neurons overlap; thus, they both discharge significantly earlier than VB cells during the slow oscillation.

In summary, our data demonstrate that VB cells discharge bursts with higher frequency and contain more action potentials than Po neurons. Slower bursts in Po compared with VPM neurons is consistent with earlier in vitro results (Landisman and Connors, 2007). Border cells were similar to VB neurons, which indicates that they may share similar intrinsic properties with first order relays. 
A
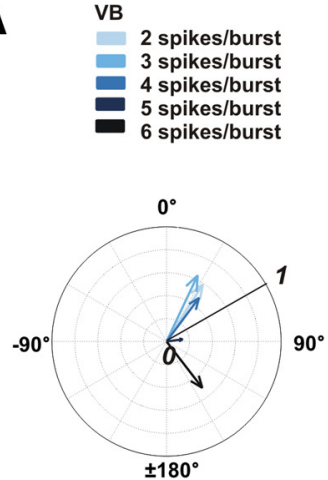

B

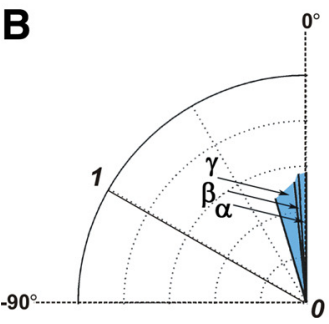

Po

2 spikes/burst

3 spikes/burst

4 spikes/burst

5 spikes/burst
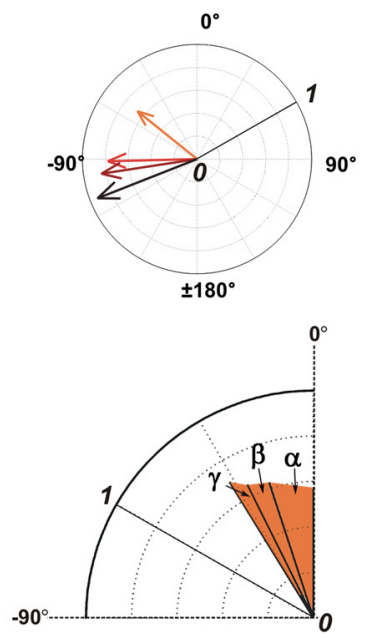

Border

2 spikes/burst

3 spikes/burst 4 spikes/burst 5 spikes/burst

6 spikes/burst

7 spikes/burst

$0^{\circ}$

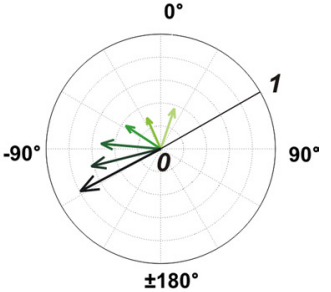

$\pm 180$

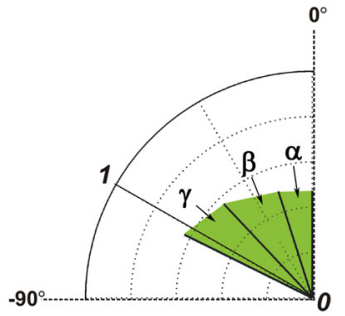

Figure 7. Phase preference of bursts with varying number of spikes in $\mathrm{VB}, \mathrm{P}_{0}$, and Border cells. $\boldsymbol{A}$, Polar plots show representative VB (blue), Po (red), and Border (green) cells. In each polar plot, arrows correspond to the phase distribution of bursts with increasing number of spikes (marked by increasing dark tone of the colors). Note phase advancement with increasing number of spikes per burst in the case of $\mathrm{Po}$ and Border cells. $\boldsymbol{B}$, Vectors showing the mean phase difference of bursts with increasing number of action potentials for VB (light blue), Po (orange), and Border (light green) neuron populations. $\alpha$ represents the mean burst phase difference of three spikes per burst and two spikes per burst; $\beta$ represents the mean phase difference of four spikes per burst and three spikes per burst; and $\gamma$ shows the phase difference of five spikes per burst and four spikes per burst. Po and Border cells fire at progressively earlier phases of the cortical up state with the increasing number of spikes in a burst, unlike VB cells.

teristics, Border cells were grouped with Po rather than VB neurons regarding phase properties.

In contrast to preferred phase, phase consistency did not differ significantly between VB and Po cells $(p=0.17)$ (Fig. $6 C, D$; supplemental Table 2, available at www.jneurosci.org as supplemental material). The mean vector length showed considerable heterogeneity within all three groups. Thus, tightly phase-locked and weakly coupled cells occurred among VB, Po, and Border neurons (Fig. 6C). Border cells were characterized by the lowest values $(p<0.001)$.

Neurons with similar phase values were not clustered spatially. We could find neurons in VB within $100 \mu \mathrm{m}$ of each other that displayed $121.83^{\circ}$ of phase difference. Similarly, in the case of two Po cells recorded $100 \mu \mathrm{m}$ apart, the difference in phase preference was $106.84^{\circ}$. These data also indicate that variability in the phase properties was not the consequence of individual differences among the animals but represents heterogeneous timing of burst generation.

\section{Phase advancement of bursts with increasing spike numbers}

The number of action potentials within a

\section{The phase of relay cell firing depends on the exact nuclear} location of the neurons

Following the characterization of bursts, we determined the preferred firing phase of relay cells relative to the ongoing cortical oscillations (phase preference) and how faithfully cells fire at their preferred phase (phase consistency) (Fig. 6). In this analysis, bursts were considered as unitary events to avoid bias caused by differences in the number of action potentials in the bursts (see Materials and Methods).

The activity of thalamic neurons in all three groups displayed phase modulation with a different amount of jitter (Fig. 6A). Phase histograms of the populations showed that relay cells, as a population, could fire at almost all phases of the slow oscillation but with clear population phase preference (Fig. $6 \mathrm{~B}$ ).

The preferred firing phase exhibited a substantial difference between the populations of $\mathrm{VB}$ and Po cells (Fig. 6C,D; supplemental Table 2, available at www.jneurosci.org as supplemental material). Most of the VB neurons (9 of 13; 69\%) displayed a preferred phase after the peak of the cortical up states $\left(0^{\circ}\right)$, whereas $80 \%$ of the Po cells (12 of 15) fired before the peak (Fig. $6 \mathrm{C})$. As a population, Po neuronal activity preceded that of $\mathrm{VB}$ cells by $49^{\circ}\left(\mathrm{VB}, 13.21^{\circ}\right.$, Po cells $-35.71^{\circ}$; difference is significant at $p<0.001$ ) (Fig. $6 D$; supplemental Table 2, available at www. jneurosci.org as supplemental material). On average, these phase values correspond to a $101 \mathrm{~ms}$ difference between the average timing of VB and Po cell activity during a single wave.

Interestingly, the mean population phase values of Border cells $(n=7)$ and Po neurons were nearly identical $\left(-35.33^{\circ}\right.$ vs $-35.71^{\circ}$ ) (Fig. 6D; supplemental Table 2, available at www. jneurosci.org as supplemental material). They showed no statistical difference from each other $(p=0.29)$, but they both differed from VB neurons $(p<0.001)$. Thus, in contrast to burst charac- thalamic burst depends on the membrane potential of the relay cells (Steriade et al., 1993a; Sherman, 2001), which is influenced by the ongoing cortical oscillation. Thus, bursts with a different number of spikes may have different phase properties. We therefore examined the correlation between the phase of bursts and the number of spikes within a burst. VB neurons did not show a systematic relationship between the number of spikes within the burst and the phase of firing $(p=0.24)$ (Fig. 7A; supplemental Table 3, available at www.jneurosci.org as supplemental material). In contrast, in Po and Border cells, bursts containing a greater number of spikes occurred at progressively earlier phases of the cortical up states (Fig. $7 A)(p<0.01$, test according to Fisher for comparison between mean angles). The average phase of bursts with three spikes per burst in Po neurons was $-18.08^{\circ}$ closer to the beginning of the up state than those with two spikes per burst (Fig. $7, \alpha$ ). This value was $-17.55^{\circ}$ in Border neurons but only $-3.29^{\circ}$ for VB cells. Similar results were obtained with bursts containing a higher number of spikes (Fig. 7B, $\beta$ and $\gamma$; supplemental Table 3, available at www.jneurosci.org as supplemental material). Thus, phase advancement with an increasing spike number was observed for Po and Border cells. VB neurons, on the other hand, did not display a systematic relationship between the number of spikes in a burst and phase.

\section{Phase relationship of relay cells to cortical oscillations in the spindle frequency range $(7-20 \mathrm{~Hz})$}

During the slow cortical oscillations recorded from S1 cortex, faster waves were frequently observed riding on the positive part of the LFP envelope (Fig. $8 \mathrm{~A}$ ), as demonstrated previously (Contreras and Steriade, 1995). These 7-20 Hz oscillations were embedded in the slow cortical up states (Fig. $8 \mathrm{~A}$ ) and were in the same frequency range as spindle oscillations (Steriade et al., 
1986). Most of the relay cells showed significant phase modulation relative to cortical oscillations in the spindle frequency range. As a population, relay cells could fire at all phases of these waves but with clear population phase preference (Fig. $8 \mathrm{~B})$. Individual cells showed heterogeneity in both phase preference and phase consistency, but most of them fired before the trough (Fig. 8C). In contrast to the slow oscillations, population data of the three groups did not show significant differences in the phase preference or consistency [test according to Fisher for comparison of mean angles, permutation test for comparison of phase consistency (see Materials and Methods); all $p>0.05$ ] (Fig. 8D; supplemental Table 4, available at www. jneurosci.org as supplemental material). These data demonstrate that, in contrast to slow oscillations, phase difference was not observed among the analyzed cell populations in the spindle frequency range.

Finally, large cell-to-cell variability was observed in the phase consistency during both the slow oscillations and spindle activity (Figs. 6, 8). Therefore, we asked whether the same cells show tight phase locking during these two types of oscillations. There was no linear correlation between the average mean vector length of the slow oscillations and the spindle waves in any of the examined anatomical groups (supplemental Fig. 3, available at www. jneurosci.org as supplemental material). Thus, we can conclude that different neurons display high phase consistency during the slow oscillations and the spindle waves, suggesting that different synaptic inputs play a role in their phase modulation.

\section{Discussion}

Our data demonstrate that temporal relationships between cortical and thalamic activity are not fixed but display dynamic changes during oscillatory activity. The timing of thalamic firing depends on the frequency of oscillation, the anatomical location of the neuron, and the precise firing pattern (Fig. 9). These data refute a simple "thalamic clock" view and indicate that the impact of thalamic activity on cortical networks changes momentarily and is continuously influenced by the ongoing oscillation.

\section{Technical considerations}

We chose ketamine/xylazine anesthesia as a model system, because it is known to produce a highly regular cortical activity. It essentially locks the variable slow-wave rhythms to a very narrow frequency range (Crunelli and Hughes, 2010). Thus, our method was biased toward homogeneity in the system. Nevertheless, we recorded variable temporal relationships between cortical and thalamic activity.

\section{Phase advancement}

Phase advancement of thalamic firing was detected in three situations: (1) as the frequency of slow oscillations increased, (2)
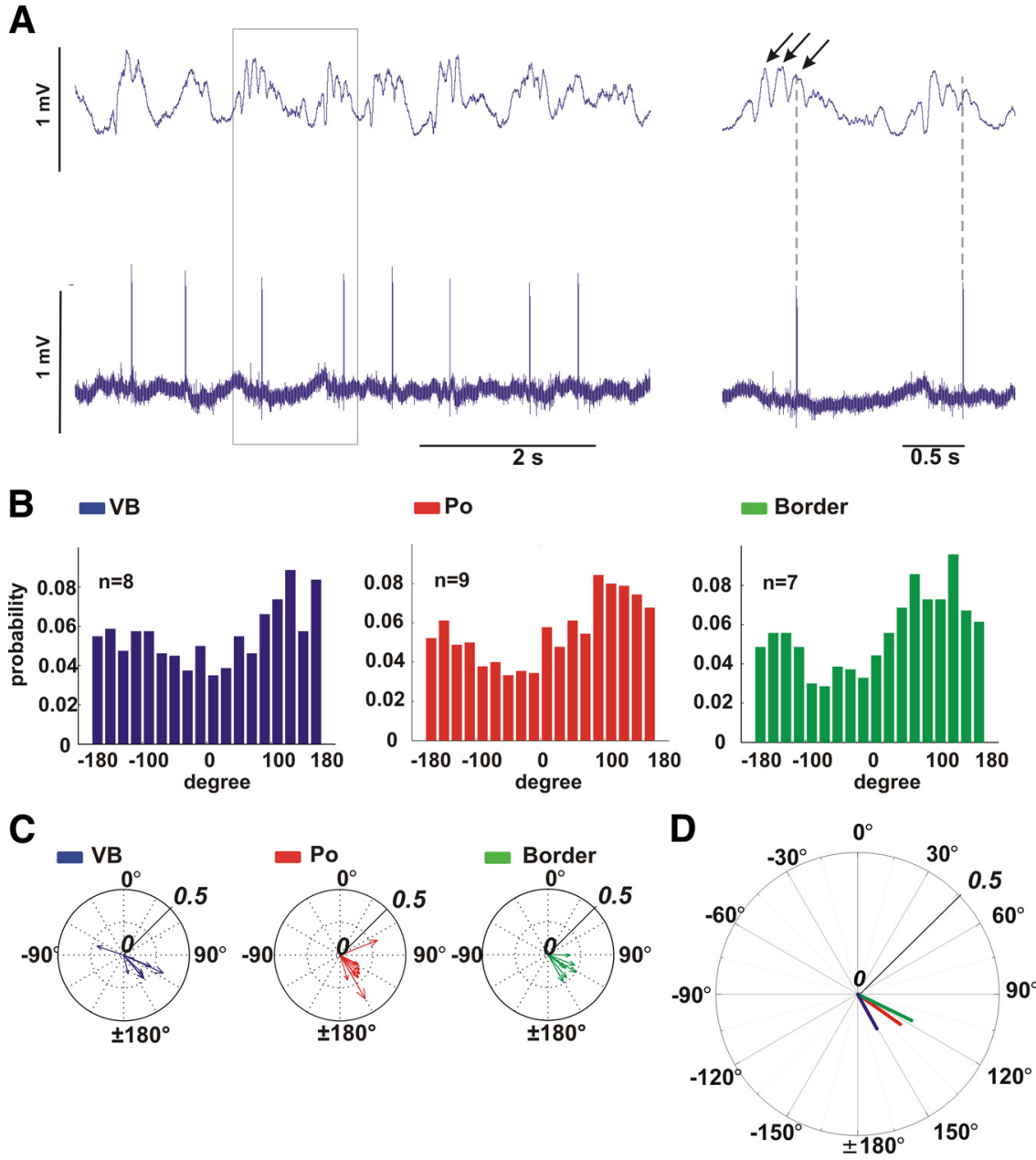

Figure 8. Phase coupling of VB, $\mathrm{P} 0$, and Border cells to $7-20 \mathrm{~Hz}$ oscillations. $A$, Simultaneous LFP recording from the $S 1$ cortex and juxtacellular recording from a thalamocortical cell (VB). Framed area of the recording is enlarged on the right. Faster oscillations in the spindle frequency range (arrows) ride on the positive part of the slow cortical oscillations. $B$, Average phase histograms (hereen). $\boldsymbol{D}$, The polar plot shows the phase preference and consistency of the three cell groups relative to $7-20 \mathrm{~Hz}$ oscillations $\left(0^{\circ}\right.$ corresponds to the positive peak). All types of cells show phase locking to cortical oscillations in the spindle oscillation range and fire preferentially before the trough of the spindle waves.

during individual faster waves embedded into a slower epoch, and (3) during bursts with a higher number of action potentials in the case of Po and Border cells. The amount of phase advancement showed cell-to-cell variability. The cause of phase advancement is presently unclear, but it probably involves an interaction between increased/faster cortical excitation and T-type calcium channel activation kinetics (Bessaïh et al., 2008). As a result of the phase advancement, thalamic firing occurred progressively earlier relative to the cortical activity with increasing frequency. The role of the thalamus and cortex in the initiation of up states is strongly debated (for review, see Crunelli and Hughes, 2010). The present results demonstrate that the timing of thalamic firing at the beginning of up states is frequency dependent. As a consequence, the thalamus has different role in the initiation and spread of up states in case of slower vs faster waves.

It is important to note that the phase change described here is qualitatively different from a similar phenomenon described in the hippocampus as "phase precession". This latter is the result of a different oscillatory cycle in pyramidal cells and the LFP and results in continuous phase advancement (O'Keefe and Recce, 1993; Harvey et al., 2009). According to our results, the phase of 
A1

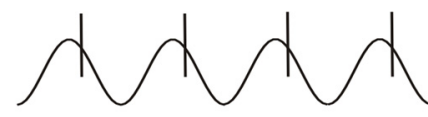

B1

A2

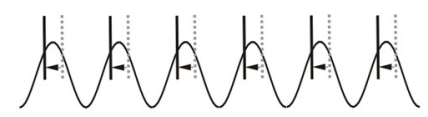

C

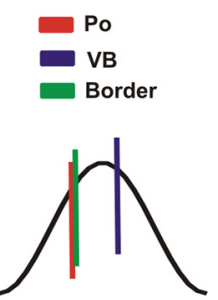

D $\quad \mathrm{P}$

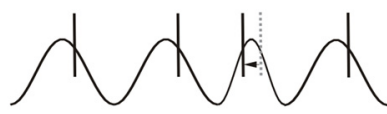

B2
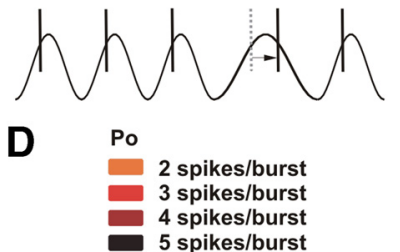

Figure 9. Schematic summary of the results demonstrating temporal and spatial phase heterogeneity in the thalamus. $\boldsymbol{A 1}, \boldsymbol{A 2}$, As the frequency of oscillation increases, the phase of a thalamocortical cell is shifted toward the beginning of the up state. $\mathbf{B 1}, \mathbf{B 2}$, The phase of the thalamocortical cell changes with each wave. A faster wave intruding into slower waves is paralleled by phase advancement (B1), whereas the appearance of a slower wave among faster ones induces immediate phase delay (B2). C, The phase is nucleus specific; Po and Border cells fire at an earlier phase than VPM cells. $\boldsymbol{D}$, Bursts with a higher number of action potentials occur progressively earlier during an up state.

thalamic activity is tightly coupled to the instantaneous frequency of cortical oscillation; hence, both phase advancement and delay can occur.

\section{Nucleus-specific timing}

VB and Po cells were active at different phases of the slow oscillation. The burst properties of VB and Po neurons were different, confirming earlier in vitro data (Landisman and Connors, 2007). Po neurons are known to receive unique sets of excitatory and inhibitory synaptic afferents, which are not present in the neighboring VB complex (Sherman and Guillery, 2006). Po cells are selectively contacted by giant excitatory terminals originating in the layer V of the S1 somatosensory cortex (Hoogland et al., 1987; Bourassa et al., 1995; Veinante et al., 2000) as well as by large multisynaptic GABAergic inputs arriving from extrareticular sources [the anterior pretectal nucleus (APT) (Bokor et al., 2005) and the zona incerta (ZI) (Barthó et al., 2002)]. Previous studies demonstrated that all of these inputs have a major impact on the firing characteristics of Po neurons (Trageser and Keller, 2004; Lavallée et al., 2005; Groh et al., 2008; Wanaverbecq et al., 2008; Theyel et al., 2010). In this study, Po neurons fired significantly earlier than VB cells during up states. In rodents and cats, layer V cortical pyramids fire at a very early phase of the up state (Sanchez-Vives and McCormick, 2000); thus, they may have an important role in driving early firing of Po cells. The strong GABAergic inputs from ZI and APT may also contribute to the early firing via the rebound mechanism (Bokor et al., 2005; Bessain et al., 2008), because the activities of both APT and ZI neurons are influenced by the cortical LFP activity (Bokor et al., 2005; Barthó et al., 2007).

Despite the fact that VB and Po cells preferred different phases during the slow oscillation, their phases were statistically indistinguishable in the spindle frequency range. The neocortex is regarded as the initiator of the slow oscillation [although recently the role of the thalamus has been revisited (see Crunelli and
Hughes, 2010)], whereas the nucleus reticularis thalami (nRT) is the major pacemaker of spindle activity (Fuentealba and Steriade, 2005). All thalamic nuclei are innervated by nRT cells (Pinault and Deschênes, 1998), and the activity of nRT cells is synchronized during spindle activity (Pinault, 2004) via gap junctions (Landisman et al., 2002) and cortical afferents (Contreras et al., 1997). Thus, during a spindle wave, VB and Po receive synchronous GABAergic input from nRT that is probably instrumental in setting the similar phase properties relative to the spindle waves. Interestingly, spindles are embedded into the slow oscillation; thus, the exact timing of the relay cell action potential is determined by the interplay of the two rhythms. If the phase difference between the VB and Po cells is translated into absolute time values, the difference $(\sim 100 \mathrm{~ms})$ is in the range of the cycle length of spindle waves. In other words, during a slow wave, early Po (and Border) cell activity is followed by VB cell firing one spindle wave later. Because thalamic nuclei can crosstalk via the nRt (Crabtree et al., 1998; Crabtree and Isaac, 2002), Po activity can actually help to recruit VB cells via the nRT using the rebound burst mechanism.

The phase consistency of VB and Po cells was found to be similar; thus, the strong input from cortical layer $\mathrm{V}$ did not induce stronger phase-locking in Po. There can be several reasons for this negative result. First, although cortical layer V cells fire at the early phase of the up states, a jitter in their activity can prevent strong phase locking of Po neurons. Indeed, our preliminary data indicate variable firing in individual cortical layer $\mathrm{V}$ cells projecting to Po (H. Bokor, personal communication). Variable extrareticular GABAergic inputs (ZI, APT) may also induce phase jitter in Po. Finally, phase advancement of bursts with a larger number of spikes in Po and Border cells also diminishes phase consistency.

\section{Border neurons}

Border cells displayed similar intrinsic features to VB neurons, but their phase properties were akin to Po cells, demonstrating that neurons with similar intrinsic properties can fire at a different phase. A likely explanation for this observation is that Border cells, by extending proximal dendrites into the Po, are in a position to receive the unique inputs of Po, and these afferents drive earlier firing similar to Po cells. It is important to note that by combining the burst features of VB cells and phase properties of Po neurons, Border cells, as a population, send a signal to the neocortex that is distinct from both the VB and Po output.

\section{Wave-to-wave modulation}

Our data indicated that the exact firing pattern of relay cells is strongly modulated in a wave-to-wave manner. The phase of firing was immediately shifted during individual slow waves displaying a frequency different from the ongoing oscillation. Furthermore, in the case of Po and Border cells, the exact phase of the burst was influenced by the number of spikes within the burst. These data suggest that phase modulation is strongly affected by the instantaneous integration of ongoing synaptic activity and that each slow wave is characterized by a specific temporal pattern of thalamocortical output.

\section{Functional implications}

According to the present results, during a cortical up state, cortical networks receive thalamic inputs in a precise temporal order. At the early phase of the up state, long bursts with a high number of spikes originating in Po and Border cells occur, followed by bursts with a lower number of spikes, with the activity of VB cells 
occurring later (Fig. 9C,D). Early thalamic firing was suggested to contribute to the manifestation and spread of slow waves (Contreras and Steriade, 1995; Rigas and Castro-Alamancos, 2007). Bursts with a high number of spikes are a very effective method of signal transmission (Swadlow and Gusev, 2001), and single Po neurons can innervate vast cortical regions, including sensory and motor areas (Deschênes et al., 1998). Thus, we argue that early Po firing can cause significant synchronous depolarization in widespread cortical regions at the early stage of up states, especially at higher frequencies, when thalamic activity is shifted to earlier phases. This population of depolarized cortical neurons could then be activated by the subsequent inputs from VB, which target single barrels in cortical layer IV. In other words, the decoupled activity of first and higher order thalamic neurons may help to establish the sequence of cortical unit activity during an up state. A similar scenario has been suggested earlier for faster cortical oscillations (Llinas et al., 2002). This demonstrates that thalamic nuclei with different cortical connectivities are recruited in distinct phases during a slow wave. This sequence defines spatial and temporal constraints for the integration of thalamic inputs from different sources in somatosensory cortex.

\section{References}

Barthó P, Freund TF, Acsády L (2002) Selective GABAergic innervation of thalamic nuclei from zona incerta. Eur J Neurosci 16:999-1014.

Barthó P, Slézia A, Varga V, Bokor H, Pinault D, Buzsáki G, Acsády L (2007) Cortical control of zona incerta. J Neurosci 27:1670-1681.

Bessaïh T, Leresche N, Lambert RC (2008) T current potentiation increases the occurrence and temporal fidelity of synaptically evoked burst firing in sensory thalamic neurons. Proc Natl Acad Sci U S A 105:11376-11381.

Bokor H, Frère SG, Eyre MD, Slézia A, Ulbert I, Lüthi A, Acsády L (2005) Selective GABAergic control of higher-order thalamic relays. Neuron 45:929-940.

Borhegyi Z, Varga V, Szilágyi N, Fabo D, Freund TF (2004) Phase segregation of medial septal GABAergic neurons during hippocampal theta activity. J Neurosci 24:8470-8479.

Bourassa J, Pinault D, Deschênes M (1995) Corticothalamic projections from the cortical barrel field to the somatosensory thalamus in rats: a single-fibre study using biocytin as an anterograde tracer. Eur J Neurosci $7: 19-30$.

Buzsáki G (1991) The thalamic clock: emergent network properties. Neuroscience 41:351-364.

Contreras D, Steriade M (1995) Cellular basis of EEG slow rhythms: a study of dynamic corticothalamic relationships. J Neurosci 15:604-622.

Contreras D, Steriade M (1997) Synchronization of low-frequency rhythms in corticothalamic networks. Neuroscience 76:11-24.

Contreras D, Destexhe A, Sejnowski TJ, Steriade M (1997) Spatiotemporal patterns of spindle oscillations in cortex and thalamus. J Neurosci 17:1179-1196.

Crabtree JW, Isaac JT (2002) New intrathalamic pathways allowing modality-related and cross-modality switching in the dorsal thalamus. J Neurosci 22:8754-8761.

Crabtree JW, Collingridge GL, Isaac JT (1998) A new intrathalamic pathway linking modality-related nuclei in the dorsal thalamus. Nat Neurosci 1:389-394.

Crunelli V, Hughes SW (2010) The slow $(<1 \mathrm{~Hz})$ rhythm of non-REM sleep: a dialogue between three cardinal oscillators. Nat Neurosci 13:9-17.

Deschênes M, Veinante P, Zhang ZW (1998) The organization of corticothalamic projections: reciprocity versus parity. Brain Res Brain Res Rev 28:286-308.

Diamond ME, von Heimendahl M, Knutsen PM, Kleinfeld D, Ahissar E (2008) "Where" and "what" in the whisker sensorimotor system. Nat Rev Neurosci 9:601-612.

Fisher N (1993) Statistical analysis of circular data. Cambridge, UK: Cambridge UP.

Fremeau RT Jr, Troyer MD, Pahner I, Nygaard GO, Tran CH, Reimer RJ, Bellocchio EE, Fortin D, Storm-Mathisen J, Edwards RH (2001) The expression of vesicular glutamate transporters defines two classes of excitatory synapse. Neuron 31:247-260.
Freund TF, Buzsáki G (1996) Interneurons of the hippocampus. Hippocampus 6:347-470.

Fuentealba P, Steriade M (2005) The reticular nucleus revisited: intrinsic and network properties of a thalamic pacemaker. Prog Neurobiol 75:125-141.

Groh A, de Kock CP, Wimmer VC, Sakmann B, Kuner T (2008) Driver or coincidence detector: modal switch of a corticothalamic giant synapse controlled by spontaneous activity and short-term depression. J Neurosci 28:9652-9663.

Harvey CD, Collman F, Dombeck DA, Tank DW (2009) Intracellular dynamics of hippocampal place cells during virtual navigation. Nature 461:941-946.

Herkenham M (1980) Laminar organization of thalamic projections to the rat neocortex. Science 207:532-535.

Hoogland PV, Welker E, Van der Loos H (1987) Organization of the projections from barrel cortex to thalamus in mice studied with Phaseolus vulgaris-leucoagglutinin and HRP. Exp Brain Res 68:73-87.

Hoogland PV, Wouterlood FG, Welker E, Van der Loos H (1991) Ultrastructure of giant and small thalamic terminals of cortical origin: a study of the projections from the barrel cortex in mice using Phaseolus vulgaris leuco-agglutinin (PHA-L). Exp Brain Res 87:159-172.

Huber R, Ghilardi MF, Massimini M, Tononi G (2004) Local sleep and learning. Nature 430:78-81.

Hughes SW, Lörincz M, Cope DW, Blethyn KL, Kékesi KA, Parri HR, Juhász G, Crunelli V (2004) Synchronized oscillations at alpha and theta frequencies in the lateral geniculate nucleus. Neuron 42:253-268.

Hurtado JM, Rubchinsky LL, Sigvardt KA (2004) Statistical method for detection of phase-locking episodes in neural oscillations. J Neurophysiol 91:1883-1898.

Jahnsen H, Llinás R (1984) Electrophysiological properties of guinea-pig thalamic neurones: an in vitro study. J Physiol 349:205-226.

Jones E (1985) The thalamus. New York: Plenum.

Klausberger T, Somogyi P (2008) Neuronal diversity and temporal dynamics: the unity of hippocampal circuit operations. Science 321:53-57.

Land PW, Kyonka E, Shamalla-Hannah L (2004) Vesicular glutamate transporters in the lateral geniculate nucleus: expression of VGLUT2 by retinal terminals. Brain Res 996:251-254.

Landisman CE, Connors BW (2007) VPM and PoM nuclei of the rat somatosensory thalamus: intrinsic neuronal properties and corticothalamic feedback. Cereb Cortex 17:2853-2865.

Landisman CE, Long MA, Beierlein M, Deans MR, Paul DL, Connors BW (2002) Electrical synapses in the thalamic reticular nucleus. J Neurosci 22:1002-1009.

Lavallée P, Urbain N, Dufresne C, Bokor H, Acsády L, Deschênes M (2005) Feedforward inhibitory control of sensory information in higher-order thalamic nuclei. J Neurosci 25:7489-7498.

Liu XB, Honda CN, Jones EG (1995) Distribution of four types of synapse on physiologically identified relay neurons in the ventral posterior thalamic nucleus of the cat. J Comp Neurol 352:69-91.

Llinás RR, Steriade M (2006) Bursting of thalamic neurons and states of vigilance. J Neurophysiol 95:3297-3308.

Llinas RR, Leznik E, Urbano FJ (2002) Temporal binding via cortical coincidence detection of specific and nonspecific thalamocortical inputs: a voltage-dependent dye-imaging study in mouse brain slices. Proc Natl Acad Sci U S A 99:449-454.

Massimini M, Tononi G, Huber R (2009) Slow waves, synaptic plasticity and information processing: insights from transcranial magnetic stimulation and high-density EEG experiments. Eur J Neurosci 29:1761-1770.

O’Keefe J, Recce ML (1993) Phase relationship between hippocampal place units and the EEG theta rhythm. Hippocampus 3:317-330.

Paxinos G, Watson C (1998) The rat brain in stereotaxic coordinates, Ed 2. London: Academic.

Pinault D (1996) A novel single-cell staining procedure performed in vivo under electrophysiological control: morpho-functional features of juxtacellularly labeled thalamic cells and other central neurons with biocytin or neurobiotin. J Neurosci Methods 65:113-136.

Pinault D (2004) The thalamic reticular nucleus: structure, function and concept. Brain Res Brain Res Rev 46:1-31.

Pinault D, Deschênes M (1998) Projection and innervation patterns of individual thalamic reticular axons in the thalamus of the adult rat: a threedimensional, graphic, and morphometric analysis. J Comp Neurol 391: 180-203.

Rigas P, Castro-Alamancos MA (2007) Thalamocortical up states: differen- 
tial effects of intrinsic and extrinsic cortical inputs on persistent activity. J Neurosci 27:4261-4272.

Sanchez-Vives MV, McCormick DA (2000) Cellular and network mechanisms of rhythmic recurrent activity in neocortex. Nat Neurosci 3: 1027-1034.

Sherman S, Guillery R (2006) Exploring the thalamus and its role in cortical function. New York: Academic.

Sherman SM (2001) Tonic and burst firing: dual modes of thalamocortical relay. Trends Neurosci 24:122-126.

Steriade M, Domich L, Oakson G (1986) Reticularis thalami neurons revisited: activity changes during shifts in states of vigilance. J Neurosci $6: 68-81$.

Steriade M, Curró Dossi R, Contreras D (1993a) Electrophysiological properties of intralaminar thalamocortical cells discharging rhythmic $(\sim 40$ $\mathrm{HZ}$ ) spike-bursts at approximately $1000 \mathrm{HZ}$ during waking and rapid eye movement sleep. Neuroscience 56:1-9.

Steriade M, McCormick DA, Sejnowski TJ (1993b) Thalamocortical oscillations in the sleeping and aroused brain. Science 262:679-685.

Steriade M, Jones EG, McCormick DA (1997) Thalamus. Oxford: Elsevier Science.
Swadlow HA, Gusev AG (2001) The impact of "bursting" thalamic impulses at a neocortical synapse. Nat Neurosci 4:402-408.

Theyel BB, Llano DA, Sherman SM (2010) The corticothalamocortical circuit drives higher-order cortex in the mouse. Nat Neurosci 13:84-88.

Trageser JC, Keller A (2004) Reducing the uncertainty: gating of peripheral inputs by zona incerta. J Neurosci 24:8911-8915.

Varga V, Hangya B, Kránitz K, Ludányi A, Zemankovics R, Katona I, Shigemoto R, Freund TF, Borhegyi Z (2008) The presence of pacemaker HCN channels identifies theta rhythmic GABAergic neurons in the medial septum. J Physiol 586:3893-3915.

Veinante P, Lavallée P, Deschênes M (2000) Corticothalamic projections from layer 5 of the vibrissal barrel cortex in the rat. J Comp Neurol 424:197-204.

Wanaverbecq N, Bodor AL, Bokor H, Slézia A, Lüthi A, Acsády L (2008) Contrasting the functional properties of GABAergic axon terminals with single and multiple synapses in the thalamus. J Neurosci 28:11848-11861.

Yu C, Derdikman D, Haidarliu S, Ahissar E (2006) Parallel thalamic pathways for whisking and touch signals in the rat. PLoS Biol 4:e124. 